\title{
Looking at Alzheimer's disease from a different angle
}

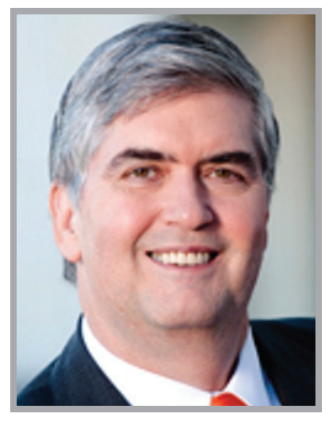

George Perry* speaks to Simi Thankaraj, Assistant Commissioning Editor: George Perry is Dean of the College of Sciences, Professor of Biology, and holds the Semmes Foundation Endowed Chair in Neurobiology at The University of Texas at San Antonio (TX, USA). Perry is recognized in the field of Alzheimer's disease research particularly for his work on oxidative stress. Perry received his Bachelor of Arts degree in Zoology with high honors from the University of California, Santa Barbara (CA, USA). After graduation, he headed to Scripps Institution of Oceanography (CA, USA) and obtained his PhD in marine biology under David Epel in 1979. He then received a postdoctoral fellowship in the Department of Cell Biology in the laboratories of Drs Bill Brinkley and Joseph Bryan at Baylor College of Medicine (TX, USA), where he laid the foundation for his observations of abnormalities in cell structures. In 1982, Perry joined the Faculty of Case Western Reserve University (OH, USA), where he currently holds an adjunct appointment. He is distinguished as one of the top Alzheimer's disease researchers, with over 1000 publications, one of the top 100 most-cited scientists in neuroscience and behavior, and one of the top 25 scientists in free radical research. Perry has been cited over 49,500-times and is recognized as a Thomson-Reuters (NY, USA) highly cited researcher. Perry is an editor for numerous journals and is the Editor-in-Chief for the Journal of Alzheimer's Disease. He is a fellow of the American Association for the Advancement of Sciences, the Microscopy Society of America, past President of the American Association of Neuropathologists, a member of the Dana Alliance for Brain Initiatives and a Fulbright Senior Specialist. Perry's research is primarily focused on how Alzheimer's disease develops and the physiological consequences of the disease at a cellular level. He is currently working to determine the sequence of events leading to damage caused by and the source of increased oxygen radicals, along with mechanisms to provide more effective treatment.

\section{Q What led to your interest in the Alzheimer's disease field?}

I was recruited for a job at Case Western Reserve University (OH, USA) to study Alzheimer's disease. Prior to then, I had never heard about Alzheimer's disease. The most surprising thing about me is that I am actually a trained marine biologist and there are some other people in the field of Alzheimer's disease who also began their training working with marine organisms. I studied sea urchin fertilization as a cell model system - I was not a cell biologist, I was simply a marine biologist who did cell biology. That's why I bring a very different talent set to studying Alzheimer's disease. I had no desire to be in the medical field, which for the USA, is very common for those entering biomedical research. I ended up getting jobs in medical schools and now I'm at a university, which is very comfortable. One of the things I realized in the last few years actually is that the training I have served me well as it is very distinctive. Alzheimer's disease as a

${ }^{*}$ College of Sciences, The University of Texas at San Antonio, One UTSA Circle, San Antonio, TX 78249, USA;

Tel.: +1 210458 4450; Fax: +1 210458 4445; george.perry@utsa.edu

Future $\because$
Medicine $\%$ part of 
field is not that close to biology; if you look at the clustering of the areas of research, it could be categorized into social biology, like psychology. It is three or more fields away from biology. What I don't understand is why people do not comprehend that whatever the body does, as a common response, has evolutionary value. A lot of ideas in medicine negate the idea of evolution, the major driving force in the natural environment. The main thing about evolution is that things are always changing to maximize their fitness; they are always trying to be better. And if we think about that concept and the concept of amyloid- $\beta$, we can see that the amyloid- $\beta$ cascade works in a completely opposite way. The other thing I had a problem with conceptually is the whole issue of genetic causality. In Alzheimer's disease, people say that genetics have proved that amyloid- $\beta$ is involved. It doesn't show causality, it shows importance. My idea is that amyloid gene linkage does not provide evidence for causality. Most people against the amyloid theory stress that amyloid is irrelevant. The more we study, the more we realize that amyloid and tau are key adjustment proteins related to the aging process, and what happens in Alzheimer's disease is the result of normal aging process failure. It fails to maintain you in a normal state. The miracle of aging is that we have all these changes that look abnormal, such as plaques and tangles, but they are needed to keep your brain healthy, at least in the earlier stages. But when the proteins change and form plaques, newer ideas of the amyloid cascade suggest that plaques are sequestering bad protein. However, amyloid could have had any structure, it didn't need to form fibers and other structures. The things that happened are selected for. You learn in college about adaptations and we think this idea should relate to Alzheimer's disease.

\section{Q What has been your greatest academic or work-related achievement to date? \\ Probably establishing the fact that oxidative stress is an important element of Alzheimer's disease.}

\section{Q What is your research focusing on at the moment?}

To understand the metabolic pathways associated with Alzheimer's disease, and what role the mitochondria play. However, what we really want to know is the transition stage from being a normal healthy individual to one who is suffering from dementia.
Q How do you think your current research will aid drug development in treating

Alzheimer's disease?

I think it will be of tremendous value because the focus of Alzheimer's disease for the past 20 years has been the removal of the pathology of the disease. But our work has shown that it is highly likely that the pathology of the disease is reactionary - not passive reactionary, but reactionary and reparative. Not causative. So therefore simply removing the pathology will always lead to mixed or negative results. Our work suggests that we need to understand and know more about the initial events, which have a major effect on the public health standpoint. To support that, there have been conclusive results in the last 5-10 years expressing how important lifestyle is in the formation of Alzheimer's disease.

\section{Q What kind of lifestyle changes do you} think someone could make in order to prevent Alzheimer's disease?

Well yes, prevention is the focus, but after you have the disease, it is less clear what lifestyle changes you would need to make. Basically, to prevent, the best type of lifestyle would be one to follow if you wanted to reduce the chance of heart disease. So I would say concentrating on consuming a diet that is rich in nutrients and low in carbohydrates, reduced in fat and engaging in exercise, both mental and physical exercise. Social activity is very important, keeping yourself positive and socially engaged. All of those have a positive effect, in addition to taking anti-inflammatories, such as aspirin, although anti-inflammatories must be taken with caution, depending on other health factors.

\section{Q What do you feel is the most difficult aspect of researching the cytopathology of Alzheimer's disease? What area do you think needs more work?}

One of the biggest issues, not just looking at my own work, but globally, is the confusion between pathology, biology, and physiology, and understanding the role of evolution. While it sounds obscure, it actually is not. Understanding how the body repairs itself, or fails to repair itself successfully, is really important in the development of therapeutics and understanding why all those changes occur. I think we are too often looking at systems that are too small. In other words, we often dissect and reduce things into simple proteins or simple animal models or cells, and 
take it out of the context of the real organism, which is the human. The human that survives with Alzheimer's disease for 20 years. I think the area that needs more work is understanding the basic biology of the human brain, how the brain alters during the aging process in possibly subtle ways. We see pathology, the gross things, which are very obvious, but is it the real action? We need to know how the brain works; it is still a mystery for all of us. The amyloid or tau cascade seem very appealing, even to me, but when we look at them in more detail, there is little direct support for them.

\section{Q What is the biggest advancement you have seen while working in the field of Alzheimer's disease?}

That would be hard to answer, as they have been many but none of them have been Earth changing. They have all been very incremental.

If I look back 30 years, finding genetic aspects of the disease certainly had a lot of promise but in reality it has told us very little about the disease. It told us that amyloid was important, but that was clear in 1906 when the disease was first described. I think the most striking thing is the whole issue of lifestyle being so important and this also holds a lot of promise. It holds promise of hope to people who are old and at a high random chance of suffering from dementia and becoming a burden on their family. Something I have seen over the last couple of years are not scientific breakthroughs, but changes in perception - I have seen these as a great improvement, families now are having more hope. In terms of new areas that people have been studying in Alzheimer's disease over the last 30 years, I think the only one is oxidative stress, as I remember all the other areas being present when I entered the profession in 1982. I guess theories about synapses were made in the 1980s but in fact, the evidence for that is appealing but equivocal.

Q You are an editor for numerous journals $\&$ the President of the Southwestern \& Rocky Mountain Division of American Association for the Advancement of Sciences \& past President of the American Association of Neuropathologists. How do you balance your time with your various responsibilities?

I actually don't know. I just manage to do it; I'm probably on the borderline of being overcommitted. I work every minute, even the weekends. When I get up in the morning, I check my emails on my mobile phone, take my dogs for a walk and come back home for breakfast, go to work and read some journals, read reports, check more emails and have an average $5 \mathrm{~h}$ of meetings. I also have evening social gatherings associated with work three- or four-times a week. In between the meetings, I confer with collaborators throughout the world. What I have noticed is that each time I took on more work I became more efficient; I actually became more productive. My primary job is being a dean of a college of over 5000 wonderful students; I love every one of them. To balance a lot of things, you need to be interested in what you do ultimately. Learn from others and figure out what works for you.

\section{Q What are you most excited to work on in} the future?

Like I mentioned earlier, I think it would be to understand the transition from normality to Alzheimer's disease. I think this would be quite difficult to understand as the field of Alzheimer's disease is blessed with an incredible number of talented investigators. We've got some of the smartest people out there working on it but why has it eluded them? One of the reasons I think is the focus on key abnormalities, such as genes and proteins, but we need to understand the early context of physiology and biology. It may be very difficult to understand because there are so many things changing in the systems biology approach. That's something I have undertaken in San Antonio (TX, USA), a more systematic approach to gene expression patterns and proteomics with a computerized analysis. I think that could yield some insight. Of course it could be that Alzheimer's disease is a really simple disease, caused by oxidative stress because of mitochondrial abnormalities, then our work could be central to that thesis. I am quite skeptical that it is that simple.

\section{Q How do you foresee the field progressing in the next 5 years?}

I don't see it progressing that much unfortunately, as it is too focused on amyloid and amyloid pathology. For a couple reasons, the original idea is dead, its reincarnation is dead, but it keeps reappearing in different formats.

\section{Q What major challenges need to be faced?}

I think until there is a truly good substitute I don't see it changing, just criticizing the amyloid approach, which Mark A Smith and I have been doing for the past 15 years, does not change the 
debate. I don't think anything's changed. Contrary ideas are published in good journals but I don't think we can change things a great deal until we can focus on oxidative stress and mitochondrial dysfunction as a substitute, but it's not simplistic or linear enough for people to accept it. People want something that has a direct therapeutic target and the amyloid hypothesis did that. It proposed that the amyloid was causative and if you removed it, then voila, everybody's fine. But when we removed the amyloid, then there was no voila, patients got slightly worse; that should have been enough to remove the issue. Now people should have gone back and defined how that wasn't the correct approach. We dealt with that aspect when we studied oxidative stress. I studied oxidative stress as a student in the mid1970s; if it was simply oxidative stress, by the old definition, then taking vitamin $\mathrm{E}$ and $\mathrm{C}$ supplements would have eradicated the disease, but it didn't work. We now know that oxidative stress is essential for life and if we were to remove it, it would be deleterious, and so taking high levels of antioxidants actually messed things up. I think that the thing with amyloid is that removing amyloid actually makes things worse, so both the theory of amyloid and oxidative stress have much in common.

\section{Disclaimer}

The opinions expressed in this interview are those of the interviewee and do not necessarily reflect the views of Future Medicine Ltd.

Financial \& competing interests disclosure

$G$ Perry has no relevant affliations or financial involvement with any organization or entity with a financial interest in or financial conflict with the subject matter or materials discussed in the manuscript. This includes employment, consultancies, honoraria, stock ownership or options, expert testimony, grants or patents received or pending, or royalties.

No writing assistance was utilized in the production of this manuscript. 